\title{
Acid Fibroblast Growth Factor and Peripheral Nerve Grafts Regulate Th2 Cytokine Expression, Macrophage Activation, Polyamine Synthesis, and Neurotrophin Expression in Transected Rat Spinal Cords
}

\author{
Huai-Sheng Kuo, ${ }^{1}$ May-Jywan Tsai, ${ }^{1}$ Ming-Chao Huang, ${ }^{1}$ Chuan-Wen Chiu, ${ }^{1}$ Ching-Yi Tsai, ${ }^{1}$ Meng-Jen Lee, ${ }^{1,3}$ \\ Wen-Cheng Huang, ${ }^{1}$ Yi-Lo Lin, ${ }^{1}$ Wen-Chun Kuo, ${ }^{1}$ and Henrich Cheng ${ }^{1,2,4,5}$ \\ ${ }^{1}$ Neural Regeneration Laboratory and ${ }^{2}$ Center for Neural Regeneration, Department of Neurosurgery, Neurological Institute, Taipei Veterans General \\ Hospital, Taipei, Taiwan, Republic of China, ${ }^{3}$ Graduate Institute of Biotechnology, Chaoyang University of Technology, Taichung, Taiwan, Republic of \\ China, and ${ }^{4}$ Department and Institute of Pharmacology and ${ }^{5} \mathrm{Faculty}$ of Medicine, National Yang-Ming University, Taipei, Taiwan, Republic of China
}

\begin{abstract}
Spinal cord injury elicits an inflammatory response that recruits macrophages to the injured spinal cord. Quantitative real-time PCR results have shown that a repair strategy combining peripheral nerve grafts with acidic fibroblast growth factor (aFGF) induced higher interleukin-4 (IL-4), IL-10, and IL-13 levels in the graft areas of rat spinal cords compared with transected spinal cords at 10 and $14 \mathrm{~d}$. This led to higher arginase I-positive alternatively activated macrophage (M2 macrophage) responses. The gene expression of several enzymes involved in polyamine biosynthesis pathways was also upregulated in the graft areas of repaired spinal cords. The treatment induced a twofold upregulation of polyamine levels at $14 \mathrm{~d}$, as confirmed by HPLC. Polyamines are important for the repair process, as demonstrated by the observation that treatment with inhibitors of arginase I and ornithine decarboxylase attenuates the functional recoveries of repaired rats. After $14 \mathrm{~d}$, the treatment also induced the expression of neurotrophin nerve growth factor (NGF) and brain-derived neurotrophic factor (BDNF), as well as M2 macrophages within grafted nerves expressing BDNF. IL-4 was upregulated in the injury sites of transected rats that received aFGF alone compared with those that received nerve grafts alone at $10 \mathrm{~d}$. Conversely, nerve graft treatment induced NGF and BDNF expression at $14 \mathrm{~d}$. Macrophages expressing polyamines and BDNF may benefit axonal regeneration at $14 \mathrm{~d}$. These results indicate that aFGF and nerve grafts regulate different macrophage responses, and M2 macrophages may play an important role in axonal regeneration after spinal cord injury in rats.
\end{abstract}

\section{Introduction}

The failure of axons to regenerate following CNS trauma results from decreased intrinsic properties of the neurons (Fawcett, 1992; Park et al., 2008; Kadoya et al., 2009), the absence of neurotrophic factors (Markus et al., 2002), and the presence of inhibitory factors in the environment (Filbin, 2003). Spinal cord injury (SCI) elicits an inflammatory response that recruits macrophages to the injured spinal cord. Macrophages can remove myelin debris after injury and produce a mixture of growth factors, cytokines, and growth-promoting surface molecules (Fawcett and Keynes, 1990; Perry and Brown, 1992; Yin et al., 2006).

Received May 17, 2010; revised Jan. 12, 2011; accepted Jan. 21, 2011.

This work was supported by Grants V9956-001 and V9856-001 from the Taipei Veterans General Hospital (Taipei, Taiwan), Grants NSC 94-3112-B-075-002 and NSC 96-2628-B-010-002-MY3 from the National Science Council in Taiwan, and a grant from the Ministry of Education (Aim for the Top University Plan). We thank Ching Jung Chen, Dann-Ying Liou, Ya-Tzu Chen, and Wen-Chun Kuo for technical assistance. This work was assisted in part by the Division of Experimental Surgery of the Department of Surgery, Taipei Veterans General Hospital.

Correspondence should be addressed to Henrich Cheng, Center for Neural Regeneration, Department of Neurosurgery, Neurological Institute, Taipei Veterans General Hospital, No. 322 Shih-Pai Road, Sec. 2, Taipei, Taiwan 112. E-mail: hc_cheng@vghtpe.gov.tw.

DOI:10.1523/JNEUROSCI.2592-10.2011

Copyright $\odot 2011$ the authors $\quad 0270-6474 / 11 / 314137-11 \$ 15.00 / 0$
The diversity of macrophage functions is endowed by unique cytokine stimuli (for review, see Gordon, 2003; Mantovani et al., 2004; Mosser and Edwards, 2008; Martinez et al., 2009; Varin and Gordon, 2009). The "classically activated" M1 macrophages respond to the proinflammatory cytokine interferon- $\gamma($ IFN- $\gamma$ ) and acquire the capacity for killing pathogens. M1 macrophages produce nitric oxide, proinflammatory cytokines, and matrix metalloproteinases, which cause tissue damage (Ueta et al., 1998) and axonal retraction (Busch et al., 2009). In contrast, the "alternatively activated" M2 macrophages respond to interleukin-4 (IL-4) and IL-13 and are involved in tissue repair. These macrophages are relatively inept killers of intracellular pathogens (Rutschman et al., 2001). They produce several extracellular matrix proteins that may promote tissue remodeling and repair (Goerdt and Orfanos, 1999; Gratchev et al., 2001). In addition, M2 macrophages promote axonal growth and overcome inhibitory substrates (Kigerl et al., 2009). Reparative approaches using macrophages implanted into the injured spinal cord have resulted in successful axonal regrowth or functional benefit (Rapalino et al., 1998; Knoller et al., 2005). Whether these cells aggravate secondary injury or promote wound repair likely depends on the phenotypes triggered by signals in the environment. 
A

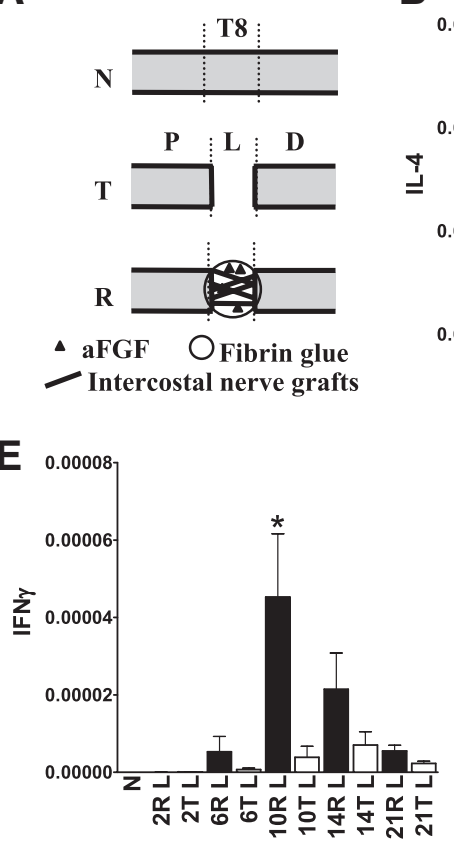

B

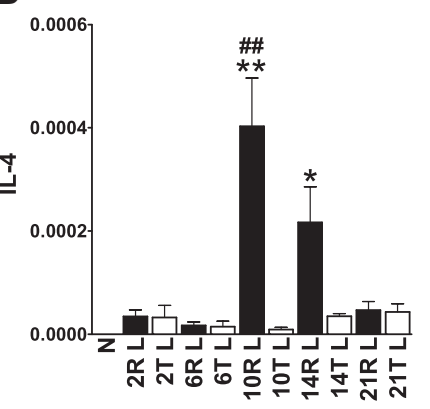

C

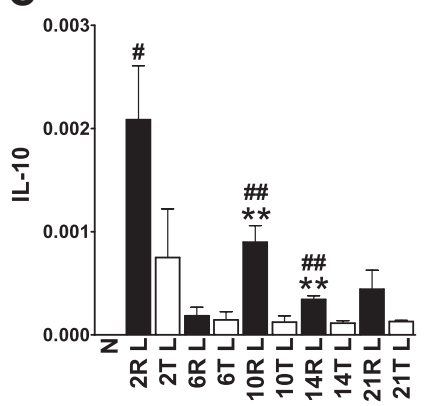

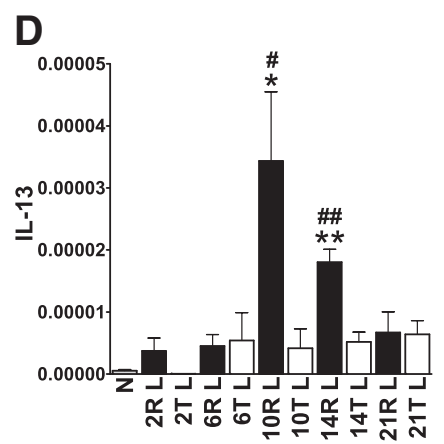

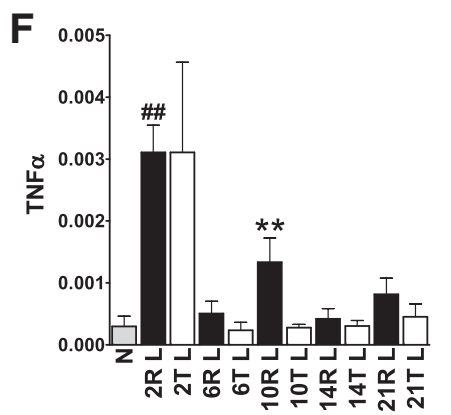

G

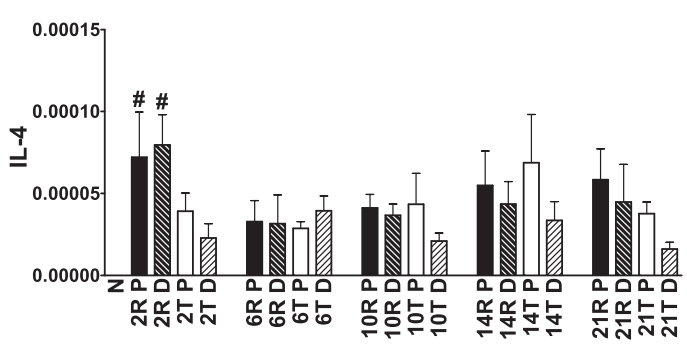

H
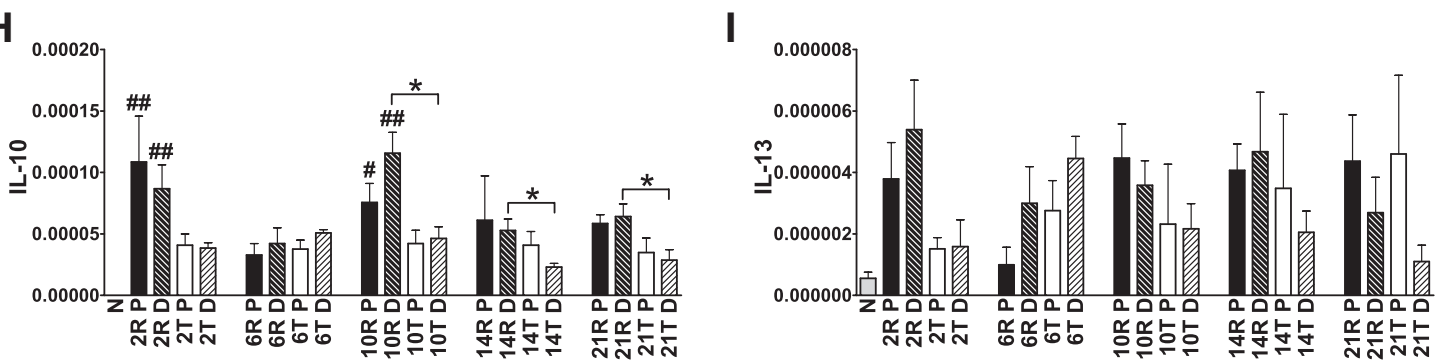

Figure 1. The expression of cytokine IL-4, IL-10, and IL-13 is upregulated in the graft areas of repaired spinal cords. $\boldsymbol{A}$, Group T, Normal rats ( $\boldsymbol{N}$ ) whose spinal cords were completely transected at T8 and for whom $5 \mathrm{~mm}$ of spinal cord tissue was removed. Group R, Spinal cord-transected rats that received a repair strategy of nerve grafts combined with aFGF in fibrin glue. P, Proximal stump; L, graft area of group R and lesion site of group T; D, distal stump. $\boldsymbol{B}-\boldsymbol{F}, \mathbf{Q}-\mathrm{PCR}$ results revealed that the expression of TNF $\alpha(\boldsymbol{C}), \mathrm{IL}-4(\boldsymbol{D}), \mathrm{IL}-10$ (E), and IL-13 (F) was transiently induced $2 \mathrm{~d}$ after injury. In contrast, the graft areas of repaired spinal cords sustained the expression of these cytokines and IFN- $\gamma(\boldsymbol{B})$. From 6 to $14 \mathrm{~d}$, significantly higher IL-10 and IL-13 levels were found in repaired spinal cords compared with normal spinal cords, and the levels of IL-4, IL-10, and IL-13 increased in the graft areas of repaired spinal cords compared with injured spinal cords. "\#p<0.05, ${ }^{\# \#} p<0.01$ for group R or T compared with the normal group by Student's $t$ test. ${ }^{*} p<0.05,{ }^{* *} p<0.01$ for group $R$ compared with group T by Student's $t$ test. Determinations are means \pm SEM from four to five experiments. G-I, IL-4 and IL-10 expression was increased in the stumps of $2 \mathrm{~d}$ repaired spinal cords compared with normal spinal cords. IL-10 expression was higher in the distal stumps of repaired spinal cords at 10 - $21 \mathrm{~d}$ than in transected spinal cords, but IL-4 and IL-13 levels were not increased in the stumps of repaired spinal cords compared with transected spinal cords. The levels in stumps were one-eighth of the levels in the graft areas at $10 \mathrm{~d} .{ }^{*} p<0.05$ between the indicated groups by Student's $t$ test. Determinations are means \pm SEM from four experiments.

An SCI repair strategy using peripheral nerve grafts and acidic fibroblast growth factor (aFGF, FGF-1) improves hindlimb locomotor function in spinal cord-transected rats (Cheng et al., 1996; Lee et al., 2002, 2004; Tsai et al., 2005). Repaired spinal cords induce the expression of the M2 macrophage marker arginase I (Arg I) 6-14 d after repair and recruited large numbers of M2 macrophages to the graft area $10 \mathrm{~d}$ after repair (Kuo et al., 2007). These findings suggest that some molecular mechanisms triggered by the repair process in the early stage of spinal cord injury are important for modulating macrophage activation and promoting axonal regeneration. To determine how aFGF and peripheral nerve grafts regulate macrophage functions, we used quantitative real-time PCR (Q-PCR) to analyze the expression of cytokines, macrophage markers, polyamine synthesis enzymes, and neurotrophins in spinal cords. Inhibitors of polyamine synthesis enzymes were used to evaluate the effect of polyamines on the functional recovery of rats in which spinal cord repair was performed. The individual effects of aFGF and nerve grafts on macrophage function were examined in spinal cord-transected rats that received either aFGF alone or nerve grafts alone.

\section{Materials and Methods}

\section{Experimental animals}

Adult female Sprague Dawley (SD) rats (225-250 g) were used in this study. All procedures involving animals were approved by the Animals Committee of Taipei Veterans General Hospital. Surgical procedures, postoperative care, and monitoring have been described previously (Cheng et al., 1996, 1997; Kuo et al., 2007). The rats were separated into several groups: group $\mathrm{N}$, normal rats $(n=4)$; group $\mathrm{T}$, spinal cordtransected rats $(n=4)$, in which the spinal cords were completely transected at $\mathrm{T} 8$ and $5 \mathrm{~mm}$ of spinal cord tissue was removed; and group R, spinal cord-transected rats that received an in vivo repair strategy developed by Cheng (Cheng et al., 1996) $(n=4-5)$. In the latter, autologous peripheral intercostal nerve segments combined with aFGF in a fibrin glue carrier were implanted to bridge the $5 \mathrm{~mm}$ gap in the transected spinal cords. The experimental designs for Groups N, T, and R are illustrated in Figure $1 A$.

Another three groups of experimental animals were used to evaluate the effect of fibrin glue, intercostal nerve grafts, and aFGF. Group U consisted of spinal cord-transected rats that received fibrin glue in the gap $(n=4)$. Group I consisted of spinal cord-transected rats that received autologous peripheral intercostal nerve grafts in fibrin glue $(n=$ 
Table 1. Primers for real-time $P C R$

\begin{tabular}{|c|c|c|c|}
\hline Gene name & GenBank accession number & Sequence & Amplicon length \\
\hline \multirow[t]{2}{*}{ Interleukin-4 } & \multirow[t]{2}{*}{ NM_201270 } & F 5'CGTCACTGACTGTAGAGAGC 3' & \multirow[t]{2}{*}{111 bp } \\
\hline & & R 5'GGGCTGTCGTTACATCCG 3' & \\
\hline Interleukin-10 & NM_012854 & F 5'CACTGCTATGTTGCCTGCTCTTAC $3^{\prime}$ & $102 \mathrm{bp}$ \\
\hline \multirow[t]{2}{*}{ Interleukin-13 } & \multirow[t]{2}{*}{ NM_053828 } & F 5' CTTGCCTTGGTGGTCTTG 3' & \multirow[t]{2}{*}{$111 \mathrm{bp}$} \\
\hline & & R 5'TCTTCTGGTCTTGTGTGATG 3' & \\
\hline Tumor necrosis factor $\alpha$ & NM_012675.3 & F 5' GCCGATTTGCCACTTCATAC $3^{\prime}$ & $200 \mathrm{bp}$ \\
\hline Interferon $\gamma$ & NM_138880 & R 5'TTCCGCTTCCTTAGGCTAGATTC 3' & $193 \mathrm{bp}$ \\
\hline \multirow[t]{2}{*}{ Arginase I } & \multirow[t]{2}{*}{ NM_007482 } & F 5'TTGATGTTGATGGACTGGAC 3' & \multirow[t]{2}{*}{$255 \mathrm{bp}$} \\
\hline & & R 5' TCTCTGGCTTATGATTACCTTC 3' & \\
\hline \multirow{2}{*}{ Agmatinase } & \multirow{2}{*}{ NM_001048185 } & F 5'CGTCAATCTCTACAACCTTCAG 3' & \multirow[t]{2}{*}{$116 \mathrm{bp}$} \\
\hline & & R 5'GATATGTGATGGTATGGTCTCC 3' & \\
\hline Ornithine decarboxylase & NM_012615.2 & F 5' CACTGTTGCTGCTGCTTCTAC 3' & $134 \mathrm{bp}$ \\
\hline Spermine synthase & NM_001033899 & R 5'ATCGCCGCCTCCCAGAATC 3' & 196 bp \\
\hline \multirow[t]{2}{*}{ Nitric oxide synthase 2} & \multirow[t]{2}{*}{ NM_012611 } & F 5'AAGAGACGCACAGGCAGAG 3' & 123 bp \\
\hline & & R 5'CAGGCACACGCAATGATGG 3' & \\
\hline Nerve growth factor & XM_227525.3 & F 5'AGCGTAATGTCCATGTTGTTCTAC 3' & $79 \mathrm{bp}$ \\
\hline & & R 5' TGCTATCTGTGTACGGTTCTGC 3' & \\
\hline Brain-derived neurotrophic factor & NM_012513 & F 5'ATTAGGTGGCTTCATAGGAGAC 3' & 178 bp \\
\hline & & R 5' GAACAGAACAGAACAGAACAGG 3' & \\
\hline Ribosomal protein L13A & NM_173340 & F 5'AGGTGGTGGTTGTACGCTGTG 3' & 102 bp \\
\hline & & R 5'GGTTGGTGTTCATCCGCTTTCG 3' & \\
\hline$\beta$-Actin & NM_031144 & F 5'ACTATCGGCAATGAGCGGTTCC 3 ' & $148 \mathrm{bp}$ \\
\hline & & R 5'CTGTGTTGGCATAGAGGTCTTTACG 3' & \\
\hline
\end{tabular}

$F$, Forward; $R$, reverse.

4). Group F consisted of spinal cord-transected rats that received aFGF in fibrin glue $(n=4)$. The experimental designs of group $\mathrm{U}, \mathrm{I}$, and $\mathrm{F}$ are illustrated in Figure 7A (see below).

To study the effects of inhibiting polyamine biosynthesis, group $\mathrm{R}$ rats were treated with either $N^{\omega}$-hydroxy-nor-L-arginine (NOHA) (Calbiochem, $10 \mu \mathrm{M}$ in fibrin glue), a competitive inhibitor of $\operatorname{Arg} \mathrm{I}(n=4)$, or DL- $\alpha$-difluoromethylornithine hydrochloride (DFMO) (Calbiochem, 10 $\mu \mathrm{M}$ in fibrin glue), an irreversible inhibitor of ornithine decarboxylase (ODC) $(n=4)$.

$R N A$ isolation, reverse transcription, and quantitative real-time PCR analysis

On specified days following injury, the rats received an overdose of sodium pentobarbital. The procedure for collecting spinal cord tissues from experimental animals for RNA isolation is illustrated in Figures $1 \mathrm{~A}$ and $7 A$. Samples designated "P" were collected from 1-cm-long spinal cord segments rostral to the injury sites, samples designated "L" were collected from the graft areas of repaired spinal cords or the lesion sites of injured spinal cords, and samples designated " $D$ " were collected from 1-cm-long spinal cord segments caudal to the injury sites. Total cellular RNA was extracted using TRIzol reagent (Invitrogen) according to the manufacturer's instructions. First-strand cDNA synthesis was primed with oligo dT and then reverse-transcribed using Superscript Reverse Transcriptase (Invitrogen), as described previously (Ma et al., 2003). cDNA levels ( $n=4-5$ per group) were quantified using primer pairs (Table 1) and a QuantiTect SYBR Green PCR kit (Qiagen) on a Light Cycler 480 (Roche Applied Science). Both ribosomal protein L13A (RPL13A) and $\beta$-actin were used as reference genes in each set of reactions, and RPL13A was used as an internal control for normalization (Jesnowski et al., 2002; Tian et al., 2007). Melt curve analyses verified the formation of the single desired PCR product. Each sample was run in duplicate. The relative differences in expressions between groups were analyzed on the basis of cycle time values using the comparative threshold cycle (Ct) method (Livak and Schmittgen, 2001), in which Ct is the cycle exhibiting the first detectable increase in SYBR Green fluorescence. The target gene quantity was normalized with a reference gene using the

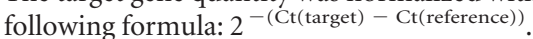

\section{Determination of polyamine concentrations}

Spinal cord segments comprising a $1 \mathrm{~cm}$ rostral stump, the lesion site, and a $1 \mathrm{~cm}$ caudal stump were collected from transected and repaired spinal cords and weighed immediately ( $n=4$ per group). The tissue was then homogenized in cold PBS containing 50\% trichloroacetic acid. Extracted fluid-containing polyamines were derivatized with benzoyl chloride, as described previously (Morgan, 1998). The benzoylated polyamines were then separated and quantified by HPLC (Waters) with a reverse-phase (C18) column (Inertsil 7 ODS-3 $4.6 \times$ $150 \mathrm{~mm}$, Vercopak) and a UV detector $(229 \mathrm{~nm})$ at room temperature. The solvent system was run isocratically using $60 \%(\mathrm{v} / \mathrm{v})$ methanol and a flow rate of $0.4 \mathrm{ml} / \mathrm{min}$. Standard curves were obtained by measuring a series of known amounts of each polyamine. Determinations used four experiments.

\section{Immunohistochemistry}

At the specified postinjury times, the rats received an overdose of sodium pentobarbital and were perfused intravascularly with $0.9 \%$ saline and 4\% paraformaldehyde in PBS. The spinal cords were collected and prepared for histological evaluation as described previously (Kuo et al., 2007). The primary antibodies used were as follows: rabbit anti-ionized calcium-binding adapter molecule-1 (Iba1) (1: 500, Wako Chemicals); mouse anti-ED1 (1:1000, Serotec); goat antiArg I (1:1000, Santa Cruz Biotechnology Inc.); mouse anti-ODC (1: 500, Sigma-Aldrich); rabbit anti-spermine (1:250, Millipore Bioscience Research Reagents); rabbit anti-spermidine (1:100, Abcam); chicken anti-brain-derived neurotrophic factor (BDNF) (1:100, R\&D Systems); mouse anti-growth-associated protein 43 (GAP-43, 1:150, Invitrogen); rabbit anti-CD86 (1:200, Abcam); and goat anti-mannose receptor (CD206, 1:200, R\&D Systems). The bound antibodies were visualized 
using the avidin-biotin-peroxidase complex (Vectastain Elite ABC kit; Vector Laboratories Inc.) with appropriate chromagens. The secondary antibodies used for fluorescence microscopy were Alexa Fluor 488 (AF488) (1:500, Invitrogen)-, AF594 (1:500, Invitrogen)-, and AF568 (1:500, Invitrogen)-conjugated secondary antibodies. Photo images were taken from stained slides of sections with a Zeiss Axioscope microscope, and the images were collected and arranged in Adobe Photoshop. Immunofluorescently labeled M1 and M2 macrophages were defined by CD86- and CD206-positive cells, respectively (Kigerl et al., 2009). The proportional areas of cells expressing either M1 or M2 antigens were quantified by NIH Image $1.44 \mathrm{~d}$ software (Wayne Rasband, National Institutes of Health, Bethesda, MD) in four longitudinal sections in the graft areas of each $14 \mathrm{~d}$ repaired rat $(n=3)$. Regenerating axons were identified by GAP-43 immunoreactivity. GAP-43-positive areas were quantified by NIH ImageJ in four longitudinal sections in the graft areas of each 6 and $14 \mathrm{~d}$ repaired rat ( $n=3$ per group).

\section{Axonal tracing}

Injections of recombinant adenoviruses result in specific and high-yield gene transfer into neurons and enable monitoring of retrograde transgene expression (Finiels et al., 1995; Han et al., 2005). Therefore, recombinant adenovirus carrying green fluorescence protein (AdGFP) was used to trace neurons, as described previously (Tsai et al., 2010a). Two weeks after repair surgery, each rat was anesthetized and received a laminectomy below the injury site. Ad-GFP (titer $=10^{6}$ pfu) was injected slowly below the grafted areas (T9) of the spinal cords using a micromanipulator. Three days after the injection, each animal was deeply anesthetized and perfused intracardially. GFP expression in the Ad-GFP-infected spinal cords was visualized using anti-mouse GFP (1:500, Abcam) coupled with Alexa Fluor488 donkey anti-mouse IgG (Invitrogen).

Regenerating neurons were traced using cholerotoxin B (CTB)-HRP (Sigma-Aldrich), as described previously (Huang et al., 2009). Three months after repair surgery, rats were anesthetized and received laminectomies above the injury sites. CTB-HRP was injected above the grafted areas (T7) of the spinal cords. Two days after the injections, the animals were deeply anesthetized with sodium pentobarbital and perfused intracardially. The spinal cords from T6 to L3 were removed for cryostat sectioning. The sections underwent reaction with diaminobenzidine (Sigma-Aldrich) before microscopic examination. The morphologies of the spinal cords were microscopically examined and recorded.

\section{Cell cultures and treatments}

Mixed neuron-glial cultures. Mixed neuron-glial cultures were prepared from the cerebrocortical regions of embryonic SD rat fetuses at $\sim 15-17$ d gestation, as described previously (Tsai et al., 2005, 2007, 2010b). Briefly, fetal cortexes were dissociated with mixtures of papain/protease/ deoxyribonuclease I $(0.1 / 0.1 / 0.03 \%)$ and plated onto poly-lysine-coated multiwell plates or insert wells (for cocultures). Cultures were maintained in DMEM (Invitrogen) supplemented with N2 (Invitrogen, for serum-free condition) or with $10 \%$ fetal bovine serum (FBS) (Invitrogen). The cells were incubated at $37^{\circ} \mathrm{C}$ in a water-saturated atmosphere of $5 \% \mathrm{CO}_{2} / 95 \%$ air.

Microglial cultures. Microglial cultures were purified from confluent mixed glial cultures. Mixed glial cultures were prepared from the neonatal cortical regions of SD rats as described previously (Tsai et al., 1996, 1997). Briefly, mechanically dissociated cortical cells were maintained in DMEM supplemented with $10 \% \mathrm{FBS}$ and incubated at $37^{\circ} \mathrm{C}$ in a watersaturated chamber. The medium was renewed every $2-3 \mathrm{~d}$. Eight to $10 \mathrm{~d}$ later, microglial cells were collected using the shake-off method (McCarthy and de Vellis, 1980) and replated onto multiwell plates. More than $98 \%$ of the cells were microglia, as identified by positive immunoreactivity for ED-1, a microglial marker.

Mixed neuron-glial cultures were maintained in serum-free medium. Cells with or without $150 \mu \mathrm{M}$ hydrogen peroxide $\left(\mathrm{H}_{2} \mathrm{O}_{2}\right)$ exposure were treated with $50 \mathrm{ng} / \mathrm{ml}$ aFGF (PeproTech), $20 \mu \mathrm{M}$ spermidine (SigmaAldrich), or $100 \mathrm{ng} / \mathrm{ml} \mathrm{BDNF}$ (PeproTech) for $48 \mathrm{~h}$. Cultures were then fixed with $4 \%$ paraformaldehye and processed for immunostaining. Neurons were stained with an antibody against the neuronal marker $\beta$ III tubulin (1:500, Covance).

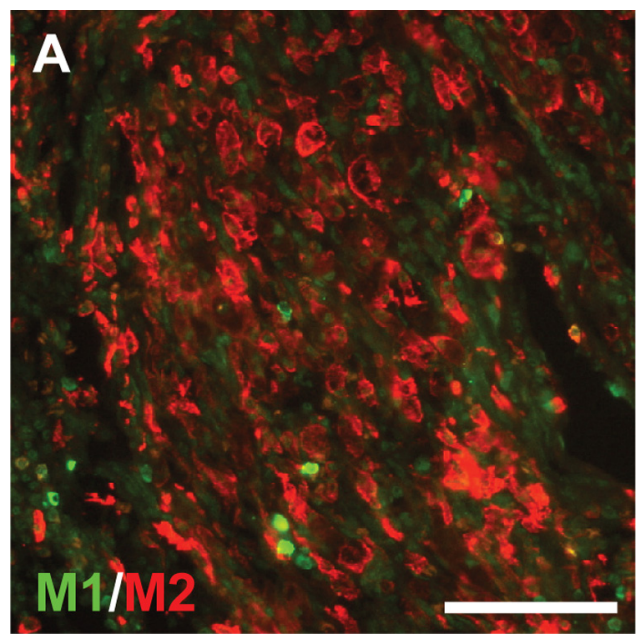

Figure 2. M2 macrophages dominate within the graft areas of $14 \mathrm{~d}$ repaired spinal cords. Sections of 14 d repaired spinal cords were stained for CD86 (M1) and mannose receptor (CD206, $M 2$ ). A large number of M2 macrophages were found in the grafted areas. Scale bar, $100 \mu \mathrm{m}$. Quantitation of macrophages expressing M1 and M2 markers in the grafted areas revealed that there were $74 \% \mathrm{M} 2$ macrophages in the grafted areas of $14 \mathrm{~d}$ repaired spinal cords ( $p<0.001$ from three rats by Student's $t$ test).

For neuron-macrophage coculture, lipopolysaccharide (LPS) (SigmaAldrich)/IFN- $\gamma$ (R\&D Systems) or IL-4 (R\&D Systems) were used to stimulate microglia differentiation into M1 or M2 phenotypes, respectively. LPS $(0.5 \mu \mathrm{g} / \mathrm{ml})$ and IFN- $\gamma(10 \mathrm{ng} / \mathrm{ml})$ or IL-4 $(10 \mathrm{ng} / \mathrm{ml})$ were added to microglia cultures for $2 \mathrm{~d}$. Then, mixed neuron-glial cultures were seeded in Transwell plates (Millicell, Millipore Corp.), transferred to microglia-seeded cultures (as cocultures), and further incubated for $2 \mathrm{~d}$. Microglial cells were stained for M1 [inducible nitric oxide synthase (iNOS), 1:400, BD Transduction Laboratories] and M2 (CD206) macrophage markers, whereas Transwell cells were stained for $\beta$ III tubulin. Immunoreactivites were quantified with NIH ImageJ $1.44 \mathrm{~d}$ software in three independent experiments.

\section{Behavioral evaluation}

The Basso, Beattie and Bresnehan (BBB) open field score was used to evaluate locomotion in terms of hindlimb functional improvement of the rats with spinal cord injuries (Basso et al., 1995). The BBB test was scored from 0 (no observable hindlimb movement) to 21 (normal hind movement) points. In this study, behavior analyses were conducted every week postsurgery until 8 weeks ( $n=4$ per group). The behavior tests were recorded by video camera, and the two examiners were blind to each behavior evaluation group.

\section{Statistical analysis}

Statistical comparisons were performed using the two-tailed Student's $t$ test. $p$-values $<0.05$ were used to indicate statistically significant differences between groups.

\section{Results}

\section{Th2 cytokines and M2 macrophages dominate the graft areas} of repaired spinal cords

To determine whether aFGF and peripheral nerve grafts provide environments that favor differentiation toward the M2 macrophage phenotype in repaired spinal cords, we examined the expression of Th1 and Th2 cytokines in spinal cord tissues using Q-PCR analysis. The expression of tumor necrosis factor $\alpha$ $(\mathrm{TNF} \alpha)$ and IL-10 displayed transient induction $2 \mathrm{~d}$ after injury and returned to a normal state after $6 \mathrm{~d}$ (Fig. 1C,E). In contrast, repaired spinal cords exhibited a second notable upregulation of all Th2 cytokine expressions after $10 \mathrm{~d}$. At 10 and $14 \mathrm{~d}$, significantly higher IL-4, IL-10, and IL-13 levels were found in the graft areas of repaired spinal cords compared with the normal group. 
A

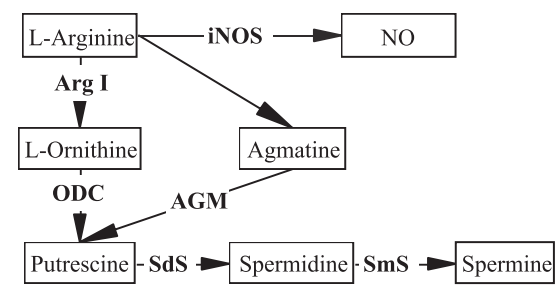

D

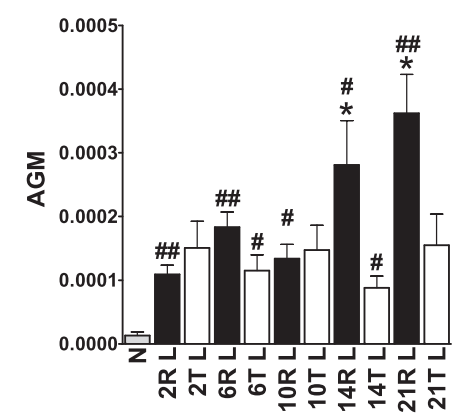

E

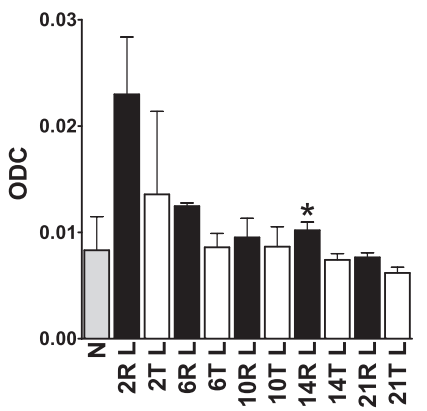

B

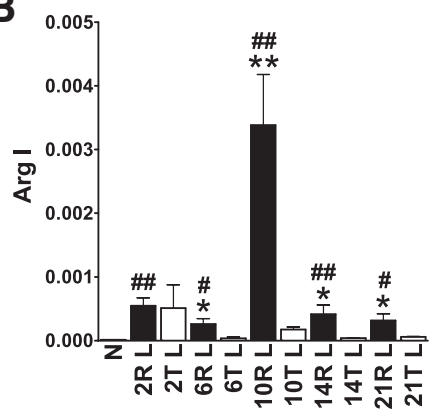

$\mathbf{F}$

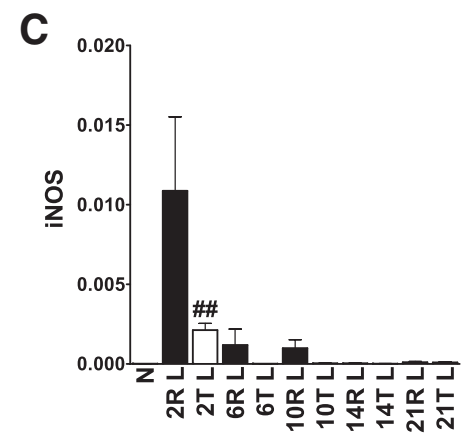

G

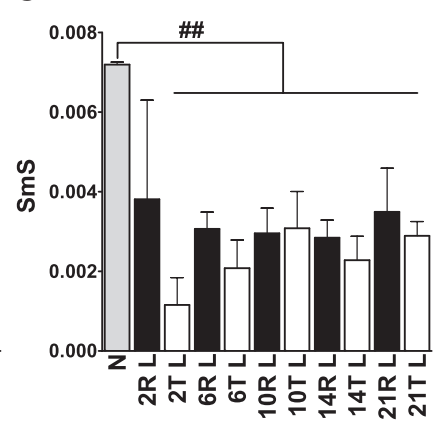

Figure 3. The expressions of $M 2$ macrophage marker Arg l and polyamine biosynthesis enzymes are upregulated in the graft areas of repaired spinal cords. $A$, Schematic drawing showing the roles of enzymes in the polyamine biosynthesis pathway. Primers for Arg I, AGM, ODC, SdS, spermine synthase (SmS), and iNOS were used for Q-PCR analysis. B-G, Repaired spinal cords maintained the expression of the M2 macrophage marker Arg I (B) rather than the M1 macrophage marker iNOS (C). Gene expression of Arg I and AGM in the graft areas was increased after injury, and expressions in group R were significantly higher than in group T from 10 to $21 \mathrm{~d}$. Gene expression of ODC, SdS, and SmS decreased after injury, but the expression of 0DC and SdS was higher in group R at 10 and $14 \mathrm{~d}$ compared with group T. ${ }^{\#} p<0.05,{ }^{\# \#} p<0.01$ of group R or T compared with the normal group by Student's $t$ test. ${ }^{*} p<0.05$, ${ }^{* *} p<0.01$ of group R compared with group T by Student's $t$ test. Determinations are means \pm SEM from four to five experiments.

In addition, all Th2 cytokines in the graft areas were higher than in injured spinal cords (Fig. 1D-F). This induction of Th2 cytokines was accompanied by a temporary upregulation of IFN- $\gamma$ and TNF $\alpha$ at $10 \mathrm{~d}$ (Fig. $1 B, C$ ). The upregulation of Th2 cytokines was prominent in the graft areas of $10 \mathrm{~d}$ repaired spinal cords, exhibiting levels in the graft areas that were approximately eight times higher than those obtained in the stumps (Fig. $1 G-I$ ).

To determine whether an increase in Th2 cytokine secretion leads to an M2 macrophage response in repaired spinal cords, the populations of M1 and M2 macrophages in $14 \mathrm{~d}$ repaired spinal cords were examined by immunostaining for M1 and M2 phenotypic markers (CD86 and mannose receptors (CD206), respectively). Large numbers of M2 macrophages were found in the grafted areas of $14 \mathrm{~d}$ repaired spinal cords (Fig. 2). Quantitation of macrophages expressing M1 and M2 markers in the grafted areas revealed that there were $74 \%$ M2 macrophages in the grafted areas of $14 \mathrm{~d}$ repaired spinal cords.

Next, we analyzed the expression of Arg I and iNOS. Q-PCR confirmed that the M2 macrophage marker Arg I was significantly higher in the graft areas of repaired spinal cords than in transected spinal cords from 6 to $21 \mathrm{~d}$ (Fig. 3B). Repaired spinal cords significantly upregulated the levels of Arg I, as opposed to the M1 macrophage marker iNOS (Fig. 3C). Immunohistochemistry demonstrated a larger number of Arg I-positive cells in the graft areas of repaired spinal cords compared with transected spinal cords at $10 \mathrm{~d}$ postinjury (supplemental Fig. $1 A, B$, available at www.jneurosci.org as supplemental material). Arg I colocalized with the Iba1-positive macrophages (supplemental Fig. $1 C$, available at www.jneurosci.org as supplemental material). Correspondingly, aFGF and peripheral nerve grafts provided environ- ments that induced Th2 cytokine expression and activated recruited macrophage differentiation toward the M2 phenotype.

\section{Upregulation of polyamine biosynthesis is necessary for functional recovery of spinal cord-repaired rats}

L-Arginine is the common enzymatic substrate of Arg I in M2 macrophages and iNOS in M1 macrophages. Arg I hydrolyzes arginine into ornithine, the first building block in the synthesis of polyamines. Using Q-PCR, we analyzed the expression profiles for a set of enzymes involved in the polyamine biosynthesis pathway (Fig. 3A). In the graft areas, gene expression of Arg I, iNOS, and agmatinase (AGM) was upregulated after injury and repair. Arg I and AGM levels in group R were significantly higher than those in group $\mathrm{T}$ from 6 to $21 \mathrm{~d}$ and 14 to $21 \mathrm{~d}$, respectively. The expression of ODC and spermidine synthase ( $\mathrm{SdS}$ ) were higher in group R at $14 \mathrm{~d}$ and $14-21 \mathrm{~d}$, respectively (Fig. 3 E, F). However, the expression of spermine synthase was decreased after injury (Fig. 3G). There was increased gene expression of the Arg I/AGM/ ODC/SdS pathway in the graft areas of repaired spinal cords. High levels of spermine immunoreactivities were also found in cells within the grafted nerves in the $10 \mathrm{~d}$ repaired spinal cords (supplemental Fig. $2 A$, available at www.jneurosci.org as supplemental material). Immunofluorescent double labeling demonstrated that many macrophages within the grafted nerves were spermine-positive (supplemental Fig. 2B,D, available at www. jneurosci.org as supplemental material) and colocalized with Arg I (supplemental Fig. $2 E, G$, available at www.jneurosci.org as supplemental material) and ODC (supplemental Fig. $2 H$, J, available at www.jneurosci.org as supplemental material). 
Q-PCR and immunohistochemistry data suggest that the upregulation of polyamine biosynthesis enzymes increases the production of polyamines in repaired spinal cord tissue. HPLC analysis of spinal cord polyamine levels confirmed this hypothesis. There is a marked and rapid increase in polyamine putrescine after injury. The induction of putrescine in injured and repaired spinal cords peaked at $2 \mathrm{~d}$ and then declined. The accumulation of putrescine in repaired spinal cords was maintained and was significantly higher than in transected spinal cords after $10 \mathrm{~d}$. The repair induced an $\sim 2$-fold upregulation of putrescine, spermidine, and spermine levels at $14 \mathrm{~d}$ compared with the transected spinal cords (Fig. 4A-C). These data demonstrate that aFGF and peripheral nerve graft treatment induces polyamine enzyme expression and polyamine production in repaired spinal cords.

Evaluation of locomotor recovery over an 8 week period demonstrated that the repair strategy provided better functional recovery among spinal cord-transected rats after 3 weeks (Fig. $4 D$ ). NOHA is a competitive inhibitor of Arg I, and DFMO is an irreversible inhibitor of ODC. Both are inhibitors of the polyamine synthesis pathway. To determine whether the improvement in locomotor recovery was dependent on this pathway, NOHA and DFMO were included in the fibrin glue when spinal cord-transected rats received aFGF and peripheral nerve grafts. NOHA- and DFMOtreated repaired rats exhibited slightly worse locomotor recovery at 3 weeks compared with the repaired animals. DFMO-treated animals exhibited significantly worse locomotor recovery after 7 weeks (Fig. 4D). We did not evaluate behavioral assessment longer than 8 weeks in this study, but in our previous studies, the motor function of repaired rats improved further from 6 to 12 months (Cheng et al., 1996). This is evidence that the activities of Arg I and ODC are important for the functional recovery of spinal cord-repaired rats and that polyamines play an important role in the repair processes.

\section{BDNF and nerve growth factor are upregulated in the graft areas of repaired spinal cords}

Macrophages express neurotrophins of the nerve growth factor (NGF) gene family and contribute to nerve regeneration and repair after injury. The expression of NGF and BDNF was analyzed by Q-PCR analysis. NGF expression was quickly upregulated after injury and repair at day 2 and was maintained at a higher level than in normal spinal cords to $21 \mathrm{~d}$. In the repaired spinal cords, NGF levels were greater than in transected spinal cords at 14 and $21 \mathrm{~d}$ (Fig. 5A). BDNF expression was slightly decreased after injury and was significantly induced in the graft areas of repaired spinal cords at 14 and $21 \mathrm{~d}$ (Fig. 5B). High immunoreactivities of BDNF were found in the grafted nerves of repaired spinal cords. At $14 \mathrm{~d}$, immunofluorescent staining of the grafted nerves of repaired spinal cords displayed Iba1-positive macrophages that were colocalized with BDNF immunoreactiv-
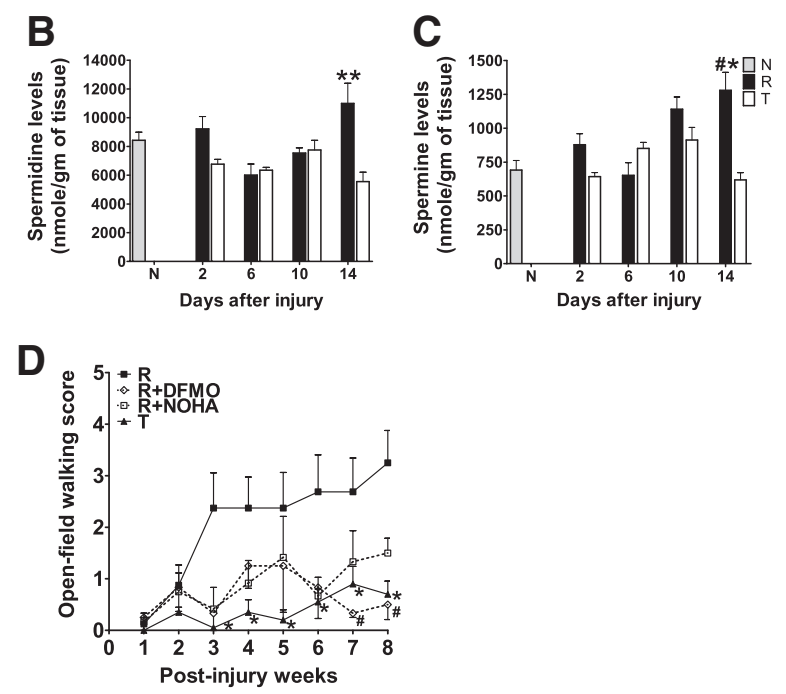

Figure 4. Polyamines are upregulated in repaired spinal cords, but inhibition of polyamine synthesis attenuates functional recovery of repaired rats. $\boldsymbol{A}-\boldsymbol{C}$, Tissues composed of proximal stumps, graft areas, and distal stumps of repaired spinal cords and thected spinal cords were collected, and polyamines were extracted. There is a marked and rapid increase in putrescine levels repaired spinal cords was maintained after $10 \mathrm{~d}$ and was significantly higher than in transected spinal cords. At $14 \mathrm{~d}$ spermidine and spermine levels in repaired spinal cords were $\sim 2$-fold higher than those in transected spinal cords. ${ }^{\#} p<0.05,{ }^{\#} p<0.01$ of group R or T compared with the normal group by Student's $t$ test. ${ }^{*} p<0.05,{ }^{* *} p<0.01$ of group R compared with group T by 8 week period, using a 21 point scale, in repaired rats (R), transected rats (T), Arg I inhibitor-treated repaired rats (NOHA), and ODC compared with repaired animals at 3 weeks. Furthermore, DFM0-treated animals exhibited worse locomotor recovery after 7 weeks. ${ }^{*} p<0.05$ of group R compared with group $\mathrm{T}$; ${ }^{\#} p<0.05$ of group R compared with R+DFM0 by Student's $t$ test. Determinations are means \pm SEM from four experiments.

ities (Fig. 5C). Arg I-positive M2 macrophages in grafted nerves also colocalized with BDNF (Fig. 5D).

\section{BDNF- and spermidine-expressing macrophages in the grafted nerves of spinal cords at $14 \mathrm{~d}$ may promote axon regeneration}

Immunostaining of GAP-43 was used to determine whether BDNFand polyamine-expressing macrophages benefit axon regeneration in repaired spinal cords. GAP-43-positive axons were mainly detected in the stumps but not in the grafted nerves of repaired spinal cords at $6 \mathrm{~d}$ (Fig. 6A). In contrast, a large number of GAP-43positive axons were found in the grafted nerves and ventral portions of the graft areas of repaired spinal cords at $14 \mathrm{~d}$ (Fig. 6B). Quantitation of proportional areas of GAP-43 immunoreactivity in the grafted areas of 6 and $14 \mathrm{~d}$ repaired spinal cords revealed that GAP-43 immunoreactivity in the grafted areas of $14 \mathrm{~d}$ repaired spinal cords was $\sim 10$ times that obtained at $6 \mathrm{~d}$ ( $p<0.01$ by Student's $t$ test). This increase in GAP-43 immunoreactivities at $14 \mathrm{~d} \mathrm{im}$ plies that these axons constitute regenerating axons rather than axons remaining in the grafted nerves. If this is the case, then axons grew into the grafted nerves and were surrounded by Iba1-positive macrophages (Fig. $6 \mathrm{C}$ ). As mentioned above, macrophages in the grafted nerves expressed BDNF and polyamines. Thus, axons seem to prefer the direction of the grafted nerves and to be accompanied by BDNF- and spermidineexpressing macrophages (Fig. $6 D, E$ ).

Two weeks after repair, recombinant Ad-GFP was injected below the grafted areas (T9) of spinal cords and used to trace neurons. Several GFP-positive regenerating axons were found along the grafted nerves and approaching the rostral stumps (Fig. $6 F-J)$. CTB-HRP labeling was used to confirm the presence of 

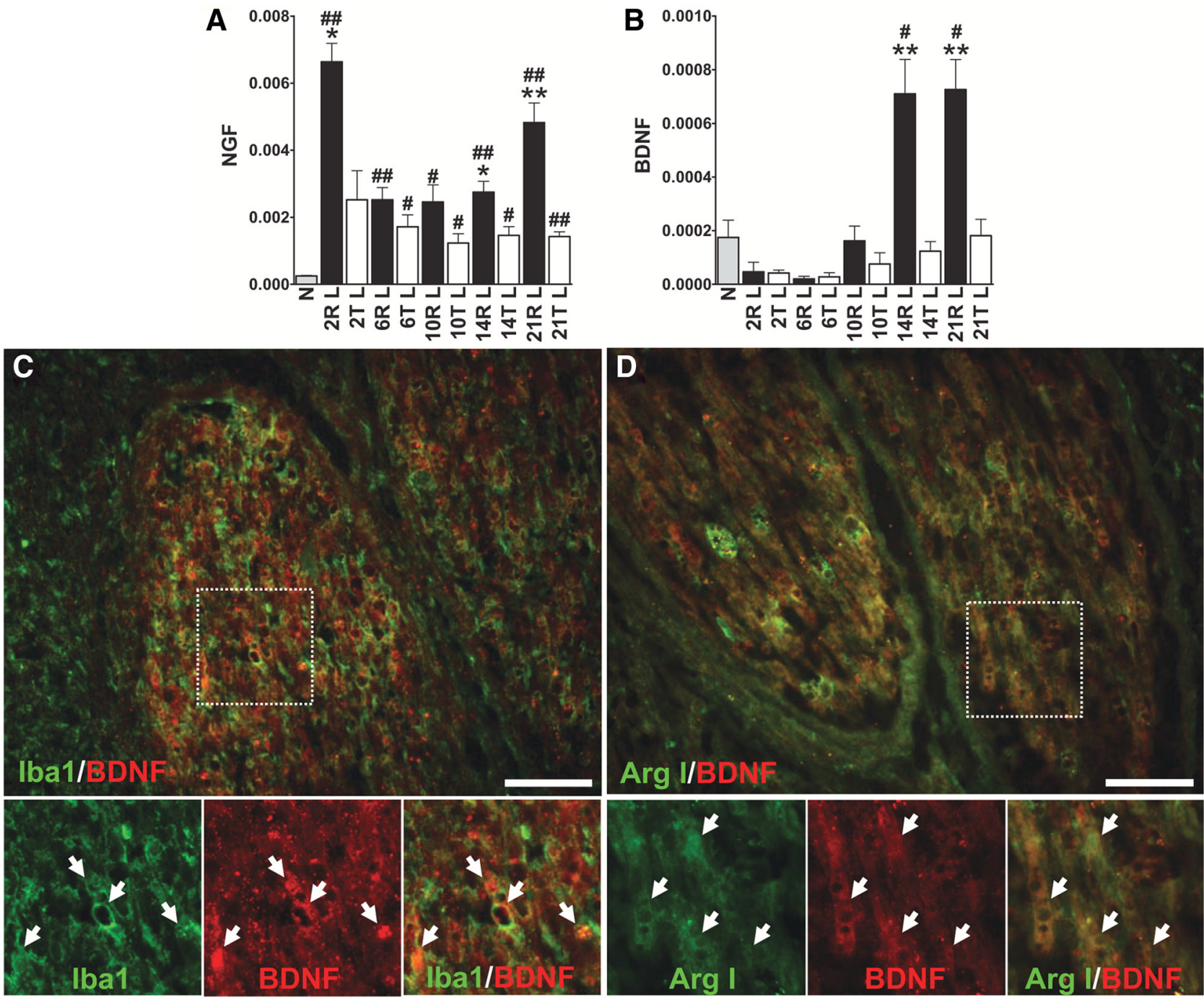

Figure 5. BDNF and NGF are upregulated in the graft areas of repaired spinal cords. $A$, NGF expression was upregulated after injury. After $6 \mathrm{~d}$, the expression was maintained at a high level from 2 to $21 \mathrm{~d}$ after repair. $\boldsymbol{B}$, BDNF expression rapidly decreased after injury. After $14 \mathrm{~d}$, expression was significantly induced in the graft areas of repaired spinal cords. ${ }^{\#} p<0.05,{ }^{\# \#} p<0.01$ of group $R$ or $T$ compared with the normal group by Student's $t$ test. ${ }^{*} p<0.05,{ }^{* *} p<0.01$ of group $R$ compared with group T by Student's $t$ test. Determinations are means \pm SEM from four to five experiments. $\boldsymbol{C}, \boldsymbol{D}$, Strong immunofluorescence of BDNF was found in the grafted nerves. Many lba1-positive macrophages $(\boldsymbol{C})$ and Arg I-positive M2 macrophages (D) in graft areas of $14 \mathrm{~d}$ repaired spinal cords colocalized with BDNF immunoreactivity. High-power magnifications of the insets in $\mathbf{C}$ and $\mathbf{D}$ are shown. Individual double-labeled cells are indicated by arrows. Scale bar, $100 \mu \mathrm{m}$.

the regenerating neurons. Three months after repair, CTB-HRP was injected above the grafted areas (T7) of the spinal cords. The presence of regenerating neurons was confirmed by the observation of CTB-HRP-positive neurons in the dorsal horns of the lumbar areas of repaired spinal cords (Fig. $6 K, L$ ). In vitro results revealed increased immunoreactivities of $\beta$ III tubulin in rat embryonic cortical neurons cocultured with M2 macrophages (supplemental Fig. 3, available at www.jneurosci.org as supplemental material). In addition, both aFGF and spermidine combined with BDNF promoted immunoreactivities of $\beta$ III in cortical neuron cultures (supplemental Fig. 4, available at www.jneurosci.org as supplemental material). Therefore, macrophages expressing polyamines and BDNF may benefit axonal regeneration.

\section{aFGF induces IL-4 and M2 macrophage marker Arg I} expression, whereas nerve grafts induce NGF and BDNF expression

Our repair strategy used aFGF and peripheral nerve grafts in fibrin glue. To explore whether the upregulation of Th2 cytokines, the M2 marker Arg I, and neurotrophins is dependent on
aFGF or nerve grafts, three additional groups of experimental animals were prepared. Group $U$ consisted of spinal cordtransected rats that received fibrin glue in the lesion site, group $\mathrm{F}$ consisted of transected rats that received aFGF in fibrin glue, and group I consisted of transected rats that received peripheral intercostal nerve grafts in fibrin glue (Fig. 7A). Q-PCR analysis revealed that aFGF alone induced higher IL-4 expression at $10 \mathrm{~d}$ than nerve grafts alone (Fig. $7 B$ ). There was a trend toward an increase in IL-4 that correlated positively with the expression of M2 marker Arg I induced by aFGF treatment (Fig. $7 B, G$ ). In contrast, aFGF had no significant effect on the expressions of IFN- $\gamma, \mathrm{TNF} \alpha$, and M1 marker iNOS (Fig. $7 E, F, H$ ). The same fibrin glue components were used in Groups R, U, F, and I, and these components may upregulate the levels of IL-10 and IL-13 (Fig. 7C,D). Meanwhile, nerve grafts distinctly induced the expression of NGF at 10 and $14 \mathrm{~d}$ compared with aFGF treatment alone (Fig. 7I). In addition, the upregulation of BDNF in the $14 \mathrm{~d}$ graft-treated spinal cords is consistent with the increase in repaired spinal cords (Fig. 7I). Therefore, nerve grafts play an im- 

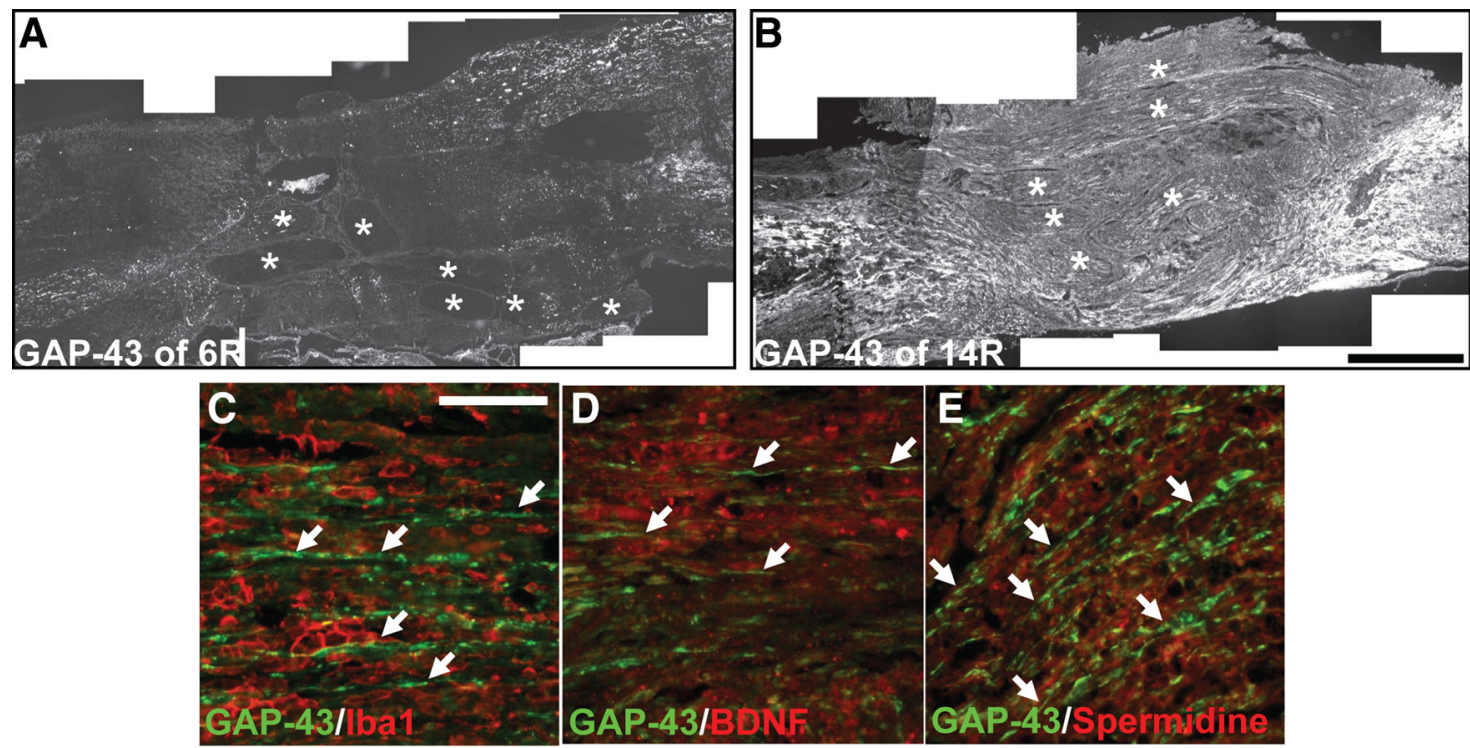

$\mathbf{F}$
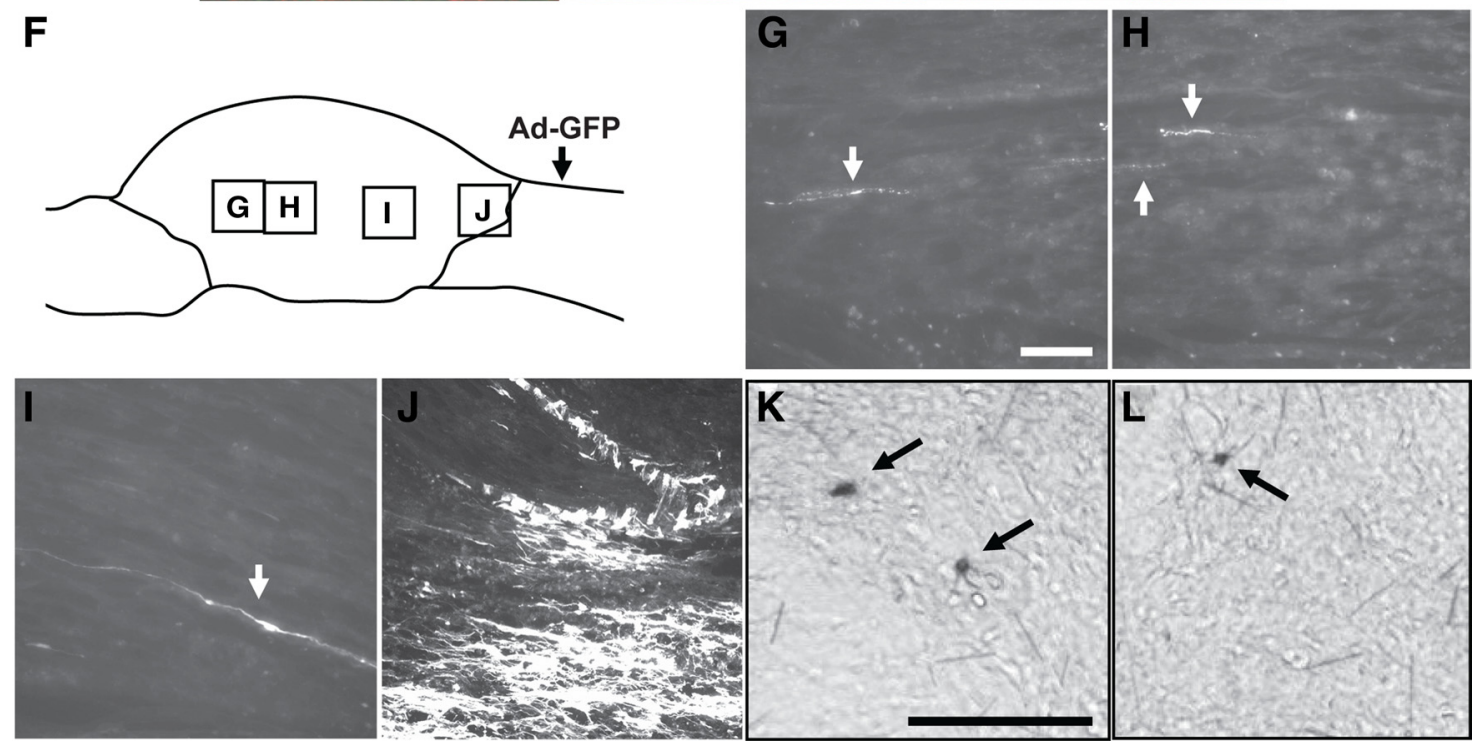

Figure 6. Regenerating axons grow into the grafted nerves of repaired spinal cords filled with BDNF- and spermidine-expressing macrophages at $14 \mathrm{~d}$. $A, B$, Longitudinal sections of $6 \mathrm{~d}(\boldsymbol{A})$ and $14 \mathrm{~d}(\boldsymbol{B})$ repaired spinal cords are positioned so that the left side of the tissue is rostral to the lesion site. Sections were stained for GAP-43. Individual grafted nerves are indicated by asterisks. Scale bar, $1 \mathrm{~mm}$. $(\boldsymbol{E}-\boldsymbol{E}$, Many GAP-43-positive axons grow alone with Iba1-positive macrophages $(\boldsymbol{C})$, BDNF-expressing macrophage $(\boldsymbol{D})$, and spermidine-expressing macrophages $(\boldsymbol{E})$ within the grafted nerves of repaired spinal cords at $14 \mathrm{~d}$. Scale bar, $100 \mu \mathrm{m}$. $\boldsymbol{F}$, Schematic representation of the recombinant Ad-GFP used to trace neurons. Two weeks after repair, Ad-GFP was injected into T9 of repaired spinal cords. G-J, There were several GFP-positive regenerating axons found along the grafted nerves and approaching the rostral stumps. Scale bar, $100 \mu \mathrm{m} . \boldsymbol{K}, \boldsymbol{L}$, Three months after repair, CTB-HRP was injected into T7 of repaired spinal cords. CTB-HRP-positive neurons were found in the dorsal horns of the lumbar areas of repaired spinal cords. Scale bar, $100 \mu \mathrm{m}$.

portant role in the upregulation of neurotrophins in repaired spinal cords after $10 \mathrm{~d}$. Toward this end, these results indicate that aFGF and nerve grafts regulate IL- 4 and neurotrophin expression independently and that a simultaneous full repair strategy produces both beneficial effects.

\section{Discussion}

Our goal was to identify the cellular and molecular changes after peripheral nerve grafts and aFGF treatment that improve hindlimb locomotor function of spinal cord-transected rats. Previous studies have demonstrated a variety of crucial functions of aFGF in the nervous system (Mason, 2007; Tsai et al., 2008), and intercostal nerve grafts have been implicated in redirecting specific pathways from white to gray matter (Cheng et al., 1996). The present study demonstrated that the repair strategy using both aFGF and nerve grafts modulates macrophage activation, poly- amine production, and neurotrophin expression, all of which are important for functional recovery. The Th status of SD rats after SCI is a mixed type of Th1 and Th2, and it is speculated that a systemic Th2 shift in the microenvironment would benefit CNS wound healing (Hendrix and Nitsch, 2007). In our full transection rat model, TNF $\alpha$ and IFN- $\gamma$ levels were increased 10- and 3.5-fold (compared to normal), respectively, $2 \mathrm{~d}$ after injury. However, the levels of Th2 cytokines and the M2 macrophage marker Arg I remained low after $6 \mathrm{~d}$, indicating a tendency toward a Th1-type response in the injury site. These results are similar to previous findings in which microglial activation was observed to peak within the lesion site 3-7 d postinjury (Popovich et al., 1997; Batchelor et al., 2008) and a differentiation of M1 macrophages is favored (Kigerl et al., 2009). In contrast to the transection model, there is a remarkable second wave of immune response after repair, in which a delayed but persistent induction 

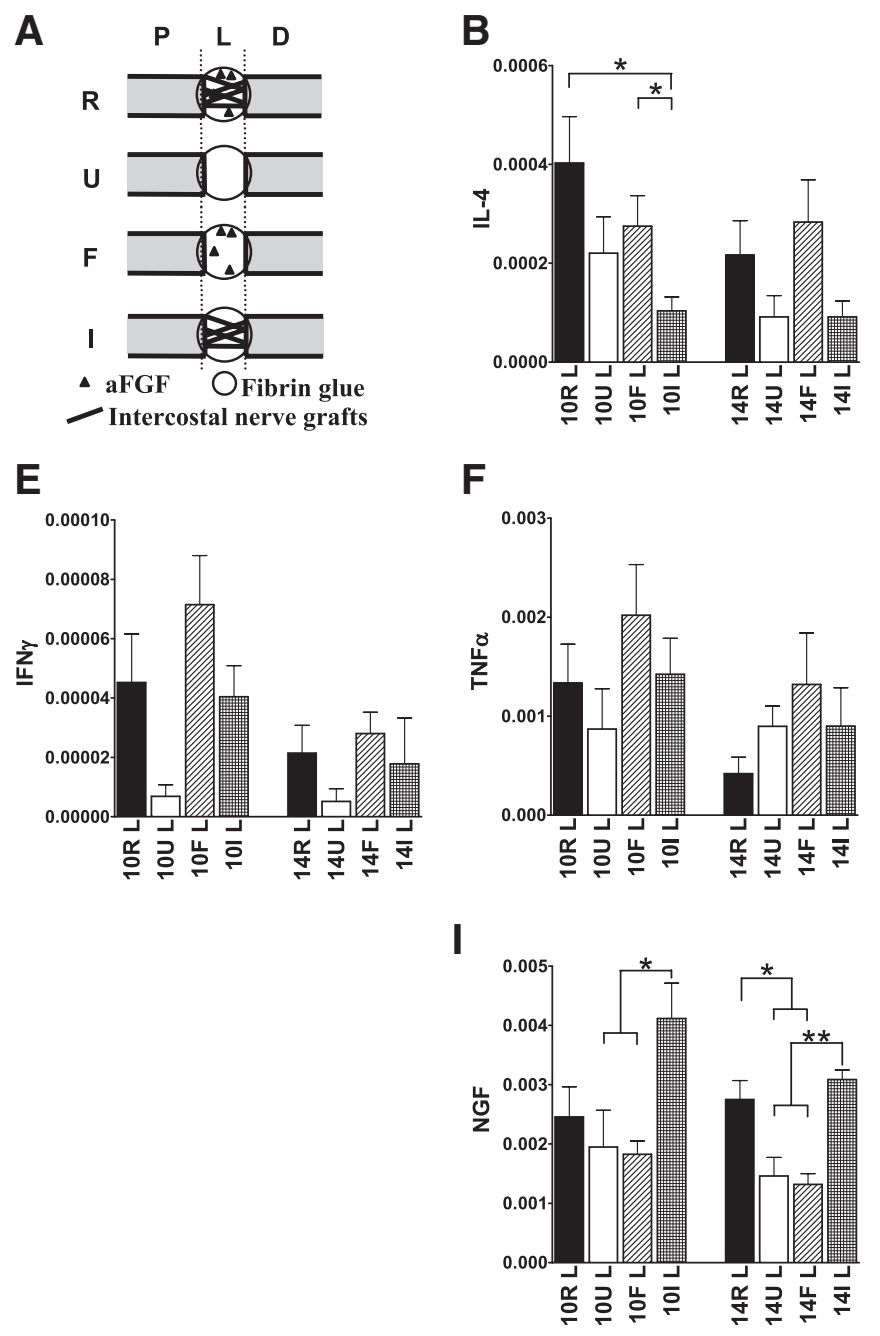
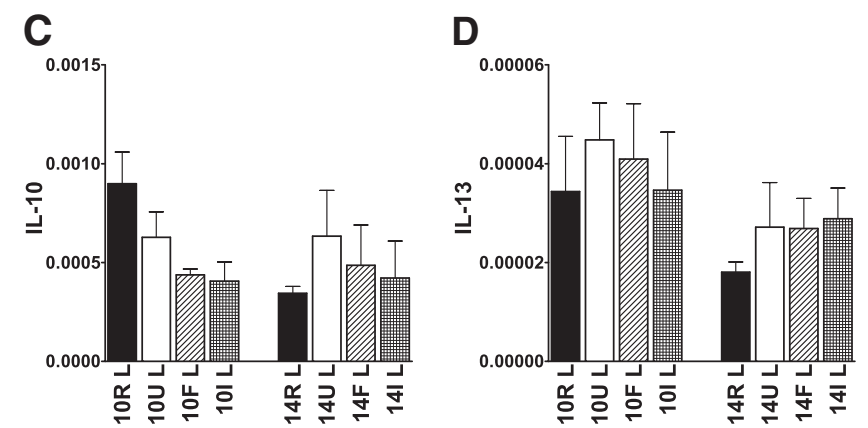

G

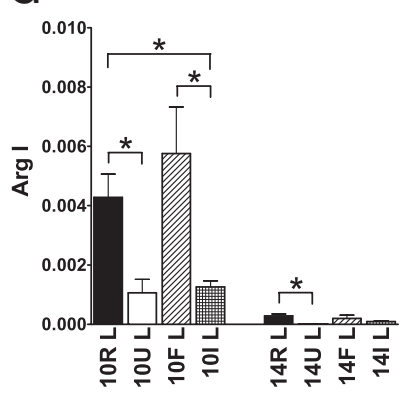

H

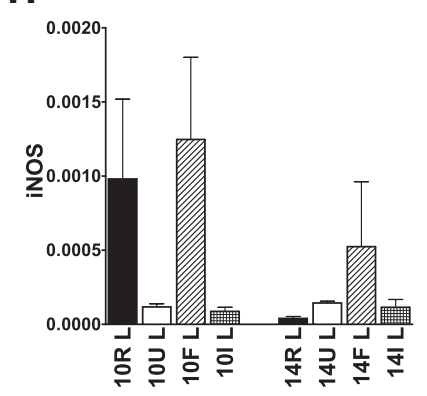

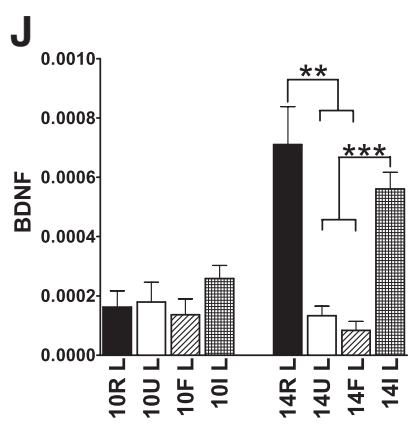

Figure 7. aFGF induces IL-4 and M2 macrophage marker Arg I expression, and nerve grafts induce BDNF and NGF expression. A, Group R consisted of spinal cord-transected rats that received a repair strategy of a nerve graft combined with aFGF in fibrin glue, group $U$ consisted of transected rats that received fibrin glue, group $F$ consisted of transected rats that received aFGF in fibrin glue, and group I consisted of transected rats that received peripheral intercostal nerve grafts in fibrin glue. $\boldsymbol{B}-\boldsymbol{J}, \mathbf{Q - P C R}$ analysis for cytokine $(\boldsymbol{B}-\boldsymbol{F}), \mathrm{M} 1$ and $M 2 \mathrm{macrophage} \mathrm{marker}(\boldsymbol{G}, \boldsymbol{H})$, and neurotrophin $(I, J)$ expression in the lesion sites of $S C I$ rats. ${ }^{*} p<0.05,{ }^{* *} p<0.01,{ }^{* * *} p<0.001$ between the indicated groups by Student's $t$ test. Determinations are means $\pm S E M$ from four experiments.

of Th2 cytokines was found. In addition, activated macrophages of the M2 phenotype were prominent around the lesion sites and graft areas in $10 \mathrm{~d}$ repaired spinal cords. A large number of M2 macrophages still dominated the graft areas in $14 \mathrm{~d}$ repaired spinal cords. Together, this Th2 shift appears to be the result of increased Th2 cytokine secretion, and this, in turn, leads to an M2 macrophage response and corresponding increase in polyamine levels in repaired spinal cords.

Previously, we proposed that aFGF may reduce neuronal cell death and inflammation after spinal cord injury (Tsai et al., 2008). In this study, we found that aFGF treatment induced Th2 cytokine IL-4 expression $10 \mathrm{~d}$ after repair and sequential upregulation of the M2 marker Arg I. The regulation of IL-4 and IL-13 and their main sources in the body are not well defined. T cells infiltrating the lesion site at 3-7 d may contribute to IL-4 expression (Popovich et al., 1997; Sroga et al., 2003; Donnelly and Popovich, 2008). Only two reports have shown that the combination of aFGF with anti-CD3 stimulates FGF receptor-1-positive T cell proliferation, but aFGF alone has no effect (Byrd et al., 1999, 2003). There have been no previous reports indicating that aFGF regulates Th2 cytokine expression. Clearly, this mechanism requires further elucidation.
Following spinal cord injury, the expression of BDNF mRNA was reported to dramatically increase (Donnelly and Popovich, 2008), with microglia, macrophages, and monocytes likely contributing to the expression of BDNF within the injury site (Batchelor et al., 1999; Dougherty et al., 2000; Ikeda et al., 2001; Batchelor et al., 2002; Bouhy et al., 2006). After 7 d, BDNF levels were reportedly lower, returning to normal levels (Ikeda et al., 2001). Our findings demonstrate a different way to regulate the expression of BDNF. Nerve grafts and full repair induced significantly higher levels of BDNF at 14 and $21 \mathrm{~d}$, and BDNF was mainly expressed by macrophages within grafted nerves. Previously, we found large numbers of activated macrophages in the injury sites of repaired spinal cords after $10 \mathrm{~d}$, and those numbers remained high for $28 \mathrm{~d}$ (Lee et al., 2008). A portion of these recruited macrophages are likely bone marrow-derived monocytes infiltrating the injury site after $7 \mathrm{~d}$ (Popovich and Hickey, 2001). When monocyte-derived macrophages interact with peripheral nerves for $1 \mathrm{~d}$, they express high levels of BDNF in vitro (Shibata et al., 2003). It is difficult to distinguish resident microglia from infiltrating monocytes. Thus, BDNF-expressing macrophages in grafted nerves may be monocytes infiltrating after $7 \mathrm{~d}$ or microglia and monocytes that require time to migrate into the 
grafted nerves, with unknown factors subsequently triggering them to express BDNF.

aFGF and nerve grafts triggered the expression of several key factors in the injury sites that can promote neuronal survival and regeneration. First, increasing Th2 cytokine levels in repaired spinal cords provides beneficial effects after injury. Adenoviralmediated IL-4 or IL-10 expression enhances the survival of axotomized retinal ganglion cells through the inhibition of $\mathrm{NO}$ synthesis (Koeberle et al., 2004). Th2 cells promote facial motoneuron survival after facial nerve axotomy, but a reduced survival rate has been reported in IL-4 knock-out mice (Deboy et al., 2006). More severe secondary inflammatory responses have been reported in IL-10 knock-out mice, resulting in worse functional recovery after spinal cord compression (Genovese et al., 2009). Moreover, treatment of contused rat spinal cords with IL-10 reportedly improves motor function recovery (Bethea et al., 1999). In addition to the known anti-inflammatory effects, IL-10 directly activates the PI3K/AKT pathway of cultured spinal cord neurons and provides a neuroprotective effect (Zhou et al., 2009). Furthermore, the expression of IL-10 by infiltrating monocytederived macrophages promotes the functional recovery of contused spinal cords in mice (Shechter et al., 2009).

Polyamines are important for axon regeneration. Treatment of cultured neurons with putrescine alone overcomes myelinassociated inhibition (Cai et al., 2002). Treatment with polyamines also promotes axon regeneration in the peripheral nerves (Gilad et al., 1996). Another in vivo study reports that spermidine can promote optic nerve regeneration (Deng et al., 2009). After spinal cord injury of rats, M1 macrophages predominate in sites of trauma and exert neurotoxic effects, but the transient M2 macrophage response promotes repair and axonal growth (Kigerl et al., 2009). Our in vitro studies revealed both coculture with M2 macrophages and treatment with spermidine combined with BDNF promoted immunoreactivities of $\beta$ III in cortical neuron cultures. We found that high levels of polyamines were synthesized in repaired spinal cords and that spermines were detected in M2 macrophages within the grafted nerves. Macrophages and/or monocytes infiltrating the injury sites after $6 \mathrm{~d}$ account for the increase in polyamine expression from 10 to $14 \mathrm{~d}$, and GAP- 43 expression is very robust within the grafted nerves at the time Arg I and polyamines are upregulated. Neuronal regeneration was confirmed by neuron tracing results. We suggest that these axons may grow from neurons in the stumps and from the supraspinal centers. The collateral sprouting should increase the chances of forming new relay circuits or activating local propriospinal neurons to improve functional recovery. The inhibition of the polyamine biosynthesis pathway appears to attenuate the functional recovery of repaired rats. Therefore, we speculate that polyamines released by these macrophages play a pivotal role in axonal regeneration.

In summary, our results indicate that aFGF induces IL- 4 expression and that nerve grafts induce NGF and BDNF expression in transected spinal cords. A full repair strategy simultaneously generates the beneficial effects of both aFGF and nerve grafts. These findings imply that M2 macrophages expressing polyamines and BDNF play an important role in axonal regeneration.

\section{References}

Basso DM, Beattie MS, Bresnahan JC (1995) A sensitive and reliable locomotor rating scale for open field testing in rats. J Neurotrauma 12:1-21.

Batchelor PE, Liberatore GT, Wong JY, Porritt MJ, Frerichs F, Donnan GA, Howells DW (1999) Activated macrophages and microglia induce dopaminergic sprouting in the injured striatum and express brain-derived neurotrophic factor and glial cell line-derived neurotrophic factor. J Neurosci 19:1708-1716.

Batchelor PE, Porritt MJ, Martinello P, Parish CL, Liberatore GT, Donnan GA, Howells DW (2002) Macrophages and microglia produce local trophic gradients that stimulate axonal sprouting toward but not beyond the wound edge. Mol Cell Neurosci 21:436-453.

Batchelor PE, Tan S, Wills TE, Porritt MJ, Howells DW (2008) Comparison of inflammation in the brain and spinal cord following mechanical injury. J Neurotrauma 25:1217-1225.

Bethea JR, Nagashima H, Acosta MC, Briceno C, Gomez F, Marcillo AE, Loor K, Green J, Dietrich WD (1999) Systemically administered interleukin-10 reduces tumor necrosis factor-alpha production and significantly improves functional recovery following traumatic spinal cord injury in rats. J Neurotrauma 16:851-863.

Bouhy D, Malgrange B, Multon S, Poirrier AL, Scholtes F, Schoenen J, Franzen R (2006) Delayed GM-CSF treatment stimulates axonal regeneration and functional recovery in paraplegic rats via an increased BDNF expression by endogenous macrophages. FASEB J 20:1239-1241.

Busch SA, Horn KP, Silver DJ, Silver J (2009) Overcoming macrophagemediated axonal dieback following CNS injury. J Neurosci 29:9967-9976.

Byrd VM, Ballard DW, Miller GG, Thomas JW (1999) Fibroblast growth factor-1 (FGF-1) enhances IL-2 production and nuclear translocation of NF-kappa B in FGF receptor-bearing Jurkat T cells. J Immunol 162:5853-5859.

Byrd VM, Kilkenny DM, Dikov MM, Reich MB, Rocheleau JV, Armistead WJ, Thomas JW, Miller GG (2003) Fibroblast growth factor receptor-1 interacts with the T-cell receptor signalling pathway. Immunol Cell Biol 81:440-450.

Cai D, Deng K, Mellado W, Lee J, Ratan RR, Filbin MT (2002) Arginase I and polyamines act downstream from cyclic AMP in overcoming inhibition of axonal growth MAG and myelin in vitro. Neuron 35:711-719.

Cheng H, Cao Y, Olson L (1996) Spinal cord repair in adult paraplegic rats: partial restoration of hind limb function. Science 273:510-513.

Cheng H, Almström S, Giménez-Llort L, Chang R, Ove Ogren S, Hoffer B, Olson L (1997) Gait analysis of adult paraplegic rats after spinal cord repair. Exp Neurol 148:544-557.

Deboy CA, Xin J, Byram SC, Serpe CJ, Sanders VM, Jones KJ (2006) Immune-mediated neuroprotection of axotomized mouse facial motoneurons is dependent on the IL-4/STAT6 signaling pathway in CD4+ T cells. Exp Neurol 201:212-224.

Deng K, He H, Qiu J, Lorber B, Bryson JB, Filbin MT (2009) Increased synthesis of spermidine as a result of upregulation of arginase I promotes axonal regeneration in culture and in vivo. J Neurosci 29:9545-9552.

Donnelly DJ, Popovich PG (2008) Inflammation and its role in neuroprotection, axonal regeneration and functional recovery after spinal cord injury. Exp Neurol 209:378-388.

Dougherty KD, Dreyfus CF, Black IB (2000) Brain-derived neurotrophic factor in astrocytes, oligodendrocytes, and microglia/macrophages after spinal cord injury. Neurobiol Dis 7:574-585.

Fawcett JW (1992) Intrinsic neuronal determinants of regeneration. Trends Neurosci 15:5-8.

Fawcett JW, Keynes RJ (1990) Peripheral nerve regeneration. Annu Rev Neurosci 13:43-60.

Filbin MT (2003) Myelin-associated inhibitors of axonal regeneration in the adult mammalian CNS. Nat Rev Neurosci 4:703-713.

Finiels F, Gimenez y Ribotta M, Barkats M, Samolyk ML, Robert JJ, Privat A, Revah F, Mallet J (1995) Specific and efficient gene transfer strategy offers new potentialities for the treatment of motor neurone diseases. Neuroreport 7:373-378.

Genovese T, Esposito E, Mazzon E, Di Paola R, Caminiti R, Bramanti P, Cappelani A, Cuzzocrea S (2009) Absence of endogenous interleukin-10 enhances secondary inflammatory process after spinal cord compression injury in mice. J Neurochem 108:1360-1372.

Gilad VH, Tetzlaff WG, Rabey JM, Gilad GM (1996) Accelerated recovery following polyamines and aminoguanidine treatment after facial nerve injury in rats. Brain Res 724:141-144.

Goerdt S, Orfanos CE (1999) Other functions, other genes: alternative activation of antigen-presenting cells. Immunity 10:137-142.

Gordon S (2003) Alternative activation of macrophages. Nat Rev Immunol 3:23-35.

Gratchev A, Guillot P, Hakiy N, Politz O, Orfanos CE, Schledzewski K, Goerdt S (2001) Alternatively activated macrophages differentially express fi- 
bronectin and its splice variants and the extracellular matrix protein betaIG-H3. Scand J Immunol 53:386-392.

Han J, Zhang Y, Chen W, Song C (2005) Monitoring retrograde adenoviral transgene expression in spinal cord and anterograde labeling of the peripheral nerves. Zhongguo Xiu Fu Chong Jian Wai Ke Za Zhi 19:215-220.

Hendrix S, Nitsch R (2007) The role of Thelper cells in neuroprotection and regeneration. J Neuroimmunol 184:100-112.

Huang MC, Lo MJ, Lin YL, Chang SE, Huang WC, Kuo WC, Tsai MJ, Kuo HS, Shih YH, Cheng H (2009) Functional recovery after the repair of transected cervical roots in the chronic stage of injury. J Neurotrauma 26:1795-1804.

Ikeda O, Murakami M, Ino H, Yamazaki M, Nemoto T, Koda M, Nakayama C, Moriya H (2001) Acute up-regulation of brain-derived neurotrophic factor expression resulting from experimentally induced injury in the rat spinal cord. Acta Neuropathol 102:239-245.

Jesnowski R, Backhaus C, Ringel J, Löhr M (2002) Ribosomal highly basic $23-\mathrm{kDa}$ protein as a reliable standard for gene expression analysis. Pancreatology 2:421-424.

Kadoya K, Tsukada S, Lu P, Coppola G, Geschwind D, Filbin MT, Blesch A, Tuszynski MH (2009) Combined intrinsic and extrinsic neuronal mechanisms facilitate bridging axonal regeneration one year after spinal cord injury. Neuron 64:165-172.

Kigerl KA, Gensel JC, Ankeny DP, Alexander JK, Donnelly DJ, Popovich PG (2009) Identification of two distinct macrophage subsets with divergent effects causing either neurotoxicity or regeneration in the injured mouse spinal cord. J Neurosci 29:13435-13444.

Knoller N, Auerbach G, Fulga V, Zelig G, Attias J, Bakimer R, Marder JB, Yoles E, Belkin M, Schwartz M, Hadani M (2005) Clinical experience using incubated autologous macrophages as a treatment for complete spinal cord injury: phase I study results. J Neurosurg Spine 3:173-181.

Koeberle PD, Gauldie J, Ball AK (2004) Effects of adenoviral-mediated gene transfer of interleukin-10, interleukin-4, and transforming growth factorbeta on the survival of axotomized retinal ganglion cells. Neuroscience 125:903-920.

Kuo HS, Tsai MJ, Huang MC, Huang WC, Lee MJ, Kuo WC, You LH, Szeto KC, Tsai IL, Chang WC, Chiu CW, Ma H, Chak KF, Cheng H (2007) The combination of peripheral nerve grafts and acidic fibroblast growth factor enhances arginase I and polyamine spermine expression in transected rat spinal cords. Biochem Biophys Res Commun 357:1-7.

Lee MJ, Chen CJ, Cheng CH, Huang WC, Kuo HS, Wu JC, Tsai MJ, Huang MC, Chang WC, Cheng H (2008) Combined treatment using peripheral nerve graft and FGF-1: changes to the glial environment and differential macrophage reaction in a complete transected spinal cord. Neurosci Lett 433:163-169.

Lee YS, Hsiao I, Lin VW (2002) Peripheral nerve grafts and aFGF restore partial hindlimb function in adult paraplegic rats. J Neurotrauma 19:1203-1216.

Lee YS, Lin CY, Robertson RT, Hsiao I, Lin VW (2004) Motor recovery and anatomical evidence of axonal regrowth in spinal cord-repaired adult rats. J Neuropathol Exp Neurol 63:233-245.

Livak KJ, Schmittgen TD (2001) Analysis of relative gene expression data using real-time quantitative PCR and the 2(-Delta Delta C(T)) Method. Methods 25:402-408.

Ma H, Xu R, Cheng H, Kuo HS, During M, Fang RH (2003) Gene transfer into human keloid tissue with adeno-associated virus vector. J Trauma 54:569-573.

Mantovani A, Sica A, Sozzani S, Allavena P, Vecchi A, Locati M (2004) The chemokine system in diverse forms of macrophage activation and polarization. Trends Immunol 25:677-686.

Markus A, Patel TD, Snider WD (2002) Neurotrophic factors and axonal growth. Curr Opin Neurobiol 12:523-531.

Martinez FO, Helming L, Gordon S (2009) Alternative activation of macrophages: an immunologic functional perspective. Annu Rev Immunol $27: 451-483$

Mason I (2007) Initiation to end point: the multiple roles of fibroblast growth factors in neural development. Nat Rev Neurosci 8:583-596.

McCarthy KD, de Vellis J (1980) Preparation of separate astroglial and oligodendroglial cell cultures from rat cerebral tissue. J Cell Biol 85:890-902.

Morgan DM (1998) Determination of polyamines as their benzoylated derivatives by HPLC. Methods Mol Biol 79:111-118.

Mosser DM, Edwards JP (2008) Exploring the full spectrum of macrophage activation. Nat Rev Immunol 8:958-969.
Park KK, Liu K, Hu Y, Smith PD, Wang C, Cai B, Xu B, Connolly L, Kramvis I, Sahin M, He Z (2008) Promoting axon regeneration in the adult CNS by modulation of the PTEN/mTOR pathway. Science 322:963-966.

Perry VH, Brown MC (1992) Macrophages and nerve regeneration. Curr Opin Neurobiol 2:679-682.

Popovich PG, Hickey WF (2001) Bone marrow chimeric rats reveal the unique distribution of resident and recruited macrophages in the contused rat spinal cord. J Neuropathol Exp Neurol 60:676-685.

Popovich PG, Wei P, Stokes BT (1997) Cellular inflammatory response after spinal cord injury in Sprague-Dawley and Lewis rats. J Comp Neurol 377:443-464.

Rapalino O, Lazarov-Spiegler O, Agranov E, Velan GJ, Yoles E, Fraidakis M, Solomon A, Gepstein R, Katz A, Belkin M, Hadani M, Schwartz M (1998) Implantation of stimulated homologous macrophages results in partial recovery of paraplegic rats. Nat Med 4:814-821.

Rutschman R, Lang R, Hesse M, Ihle JN, Wynn TA, Murray PJ (2001) Cutting edge: Stat6-dependent substrate depletion regulates nitric oxide production. J Immunol 166:2173-2177.

Shechter R, London A, Varol C, Raposo C, Cusimano M, Yovel G, Rolls A, Mack M, Pluchino S, Martino G, Jung S, Schwartz M (2009) Infiltrating blood-derived macrophages are vital cells playing an anti-inflammatory role in recovery from spinal cord injury in mice. Plos Med 6:e1000113.

Shibata A, Zelivyanskaya M, Limoges J, Carlson KA, Gorantla S, Branecki C, Bishu S, Xiong H, Gendelman HE (2003) Peripheral nerve induces macrophage neurotrophic activities: regulation of neuronal process outgrowth, intracellular signaling and synaptic function. J Neuroimmunol 142:112-129.

Sroga JM, Jones TB, Kigerl KA, McGaughy VM, Popovich PG (2003) Rats and mice exhibit distinct inflammatory reactions after spinal cord injury. J Comp Neurol 462:223-240.

Tian YF, Zhang PB, Xiao XL, Zhang JS, Zhao JJ, Kang QY, Chen XL, Qiu F, Liu Y (2007) The quantification of ADAMTS expression in an animal model of cerebral ischemia using real-time PCR. Acta Anaesthesiol Scand 51:158-164.

Tsai MC, Shen LF, Kuo HS, Cheng H, Chak KF (2008) Involvement of aFGF in spinal cord injury repair processes revealed by a proteomic approach. Mol Cell Proteomics 7:1668-1687.

Tsai MJ, Chang YF, Schwarcz R, Brookes N (1996) Characterization of L-alpha-aminoadipic acid transport in cultured rat astrocytes. Brain Res 741:166-173.

Tsai MJ, Goh CC, Wan YL, Chang C (1997) Metabolic alterations produced by 3-nitropropionic acid in rat striata and cultured astrocytes: quantitative in vitro $\mathrm{H}-1$ nuclear magnetic resonance spectroscopy and biochemical characterization. Neuroscience 79:819-826.

Tsai MJ, Shyue SK, Weng CF, Chung Y, Liou DY, Huang CT, Kuo HS, Lee MJ, Chang PT, Huang MC, Huang WC, Liou KD, Cheng H (2005) Effect of enhanced prostacyclin synthesis by adenovirus-mediated transfer on lipopolysaccharide stimulation in neuron-glia cultures. Ann N Y Acad Sci 1042:338-348.

Tsai MJ, Weng CF, Shyue SK, Liou DY, Chen CH, Chiu CW, Yang TH, Pan HA, Liao RI, Kuo HS, Huang MC, Huang WC, Hoffer BJ, Cheng H (2007) Dual effect of adenovirus-mediated transfer of BMP7 in mixed neuron-glial cultures: neuroprotection and cellular differentiation. J Neurosci Res 85:2950-2959.

Tsai MJ, Pan HA, Liou DY, Weng CF, Hoffer BJ, Cheng H (2010a) Adenoviral gene transfer of bone morphogenetic protein-7 enhances functional recovery after sciatic nerve injury in rats. Gene Ther 17:1214-1224.

Tsai MJ, Liao JF, Lin DY, Huang MC, Liou DY, Yang HC, Lee HJ, Chen YT, Chi CW, Huang WC, Cheng H (2010b) Silymarin protects spinal cord and cortical cells against oxidative stress and lipopolysaccharide stimulation. Neurochem Int 57:867-875.

Ueta E, Yoneda K, Yamamoto T, Osaki T (1998) Influence of twinline, an elemental diet, on the generation of nitric oxide and reactive-oxygen intermediates from mouse peritoneal macrophages and polymorphonuclear leukocytes. J Pharm Pharmacol 50:935-942.

Varin A, Gordon S (2009) Alternative activation of macrophages: Immune function and cellular biology. Immunobiology 214:630-641.

Yin Y, Henzl MT, Lorber B, Nakazawa T, Thomas TT, Jiang F, Langer R, Benowitz LI (2006) Oncomodulin is a macrophage-derived signal for axon regeneration in retinal ganglion cells. Nat Neurosci 9:843-852.

Zhou Z, Peng X, Insolera R, Fink DJ, Mata M (2009) Interleukin-10 provides direct trophic support to neurons. J Neurochem 110:1617-1627. 